\title{
The HST Sample of Radio-Loud Quasars: Fe II and Other Correlations
}

\author{
D. Wills, K. L. Thompson, M.S. Brotherton, and Beverley J. Wills \\ McDonald Observatory and Department of Astronomy, RLM 15.308, \\ University of Texas at Austin, TX 78712, USA
}

\section{J. A. Baldwin}

CTIO, Casilla 603, La Serena, Chile

\section{R. F. Carswell}

Institute of Astronomy, Madingley Road, Cambridge, CB3 OHA, UK

\section{W. A. Browne}

Nuffield Radio Astronomy Laboratories, Jodrell Bank, Cheshire SK11 $g D L, U K$

\section{H. Netzer}

\section{Tel-Aviv University, Tel-Aviv, Israel}

We are analyzing the emission lines and continuum spectra of a sample of $\sim 61$ radio-loud quasars to investigate dependences amongst various spectral, radio, and X-ray parameters, in particular to look for dependences of spectral properties on inclination of the the radio-jet axis (Wills et al. 1997).

We have carried out conventional correlation analyses, as well as spectral principal-component analyses (SPCA) as described in the previous paper (Wills et al. 1997). We found that the single largest contribution to the spectrum-tospectrum variations was from a component - the 'first principal component' (PC1) - that has a more UV (or less red) continuum than the mean spectrum, and a weaker narrow-line (NLR) spectrum. The 'second principal component' (PC2) accounts for only 8-12\% of the spectrum-to-spectrum variation, contributing a weaker UV, and stronger red continuum, and showing stronger narrow emission lines. There is also a tendency for the broad (BLR) lines in the second principal component spectrum to be narrower (note the sharper He II $\lambda 1640$ and [O III] $\lambda 1663$ features redward of the stronger $C$ IV $\lambda 1549$ emission line, and perhaps a narrow component of the $\mathrm{N} \vee \lambda 1240$ line redward of Ly $\alpha$ ).

We have investigated the meanings of these components by correlating their weights in individual spectra of the sample, $\mathrm{PC} 1$ and $\mathrm{PC} 2$, with other variables, and the prime results are shown in the correlation diagrams (Fig. 1).

PC1 is anti-correlated with core-dominance. Thus we speculate that this component represents a changing inclination of the radio-axis to the line-ofsight. There is indeed a negative correlation of $\mathrm{PC} 1$ with the ratio of NLR strengths: [O II $] \lambda 3727 /[\mathrm{O} I \mathrm{II}] \lambda 5007$. While it has been suggested that [O III] is more obscured in lobe-dominant quasars because the highest-ionization regions 

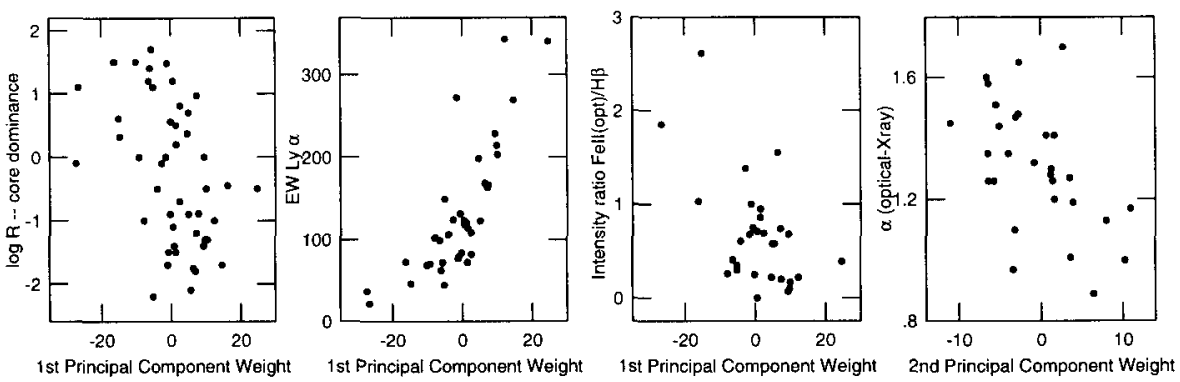

Figure 1. The prime correlations with principal component weights.

are closest to the central ionizing continuum (e.g., Hes et al. 1994, Baker et al. 1994), this trend appears to be in the opposite sense. We note that the $[\mathrm{O} \mathrm{II}] \lambda 3727 /\left[\mathrm{O}_{\mathrm{HII}}\right] \lambda 5007$ intensity ratio does not depend directly on $R$ at all. Could the increasing equivalent width of Ly $\alpha$ and decreasing intensity ratio Fe II (opt) $/ \mathrm{H} \beta$ be explained by increasing inclination?

The PC2 weights correlate significantly with the optical-to-X-ray spectral index in the sense that the redder or less-UV the continuum is, the stronger is the X-ray emission relative to the optical emission. This appears not to be an orientation effect as $\mathrm{PC} 2$ is not correlated with $R$. We speculate that this $\mathrm{PC} 2$ vs. $\alpha$ (optical-X-ray) anti-correlation may be the result of a stronger ionizing flux penetrating through the densest, highest-velocity BLR gas to the lower-density, lower-velocity, gas beyond (Brotherton 1997).

This investigation is continuing - by enlarging the radio-loud sample using $H S T$ archival and our ground-based spectra (with Chunyan Wei), as well as by detailed comparison with a sample of radio-quiet QSOs being observed with HST and from the ground (Wills, Wills, Brotherton, Laor, Wilkes, and Ferland).

Acknowledgments. We are indebted to Paul J. Francis for his continued interest and valuable discussions.

\section{References}

Baker, J.C., Hunstead, R.W., Kapahi, V.K., \& Subrahmanya, C. R. 1994, in The First Stromlo Symposium: The Physics of Active Galaxies, ed. G. V. Bicknell, M. A. Dopita, \& P. J. Quinn (San Francisco: Astronomical Society of the Pacific), 195.

Brotherton, M. S. 1997, this volume.

Hes, R., Fosbury, R. A. E., \& Barthel, P. D. 1994, in The First Stromlo Symposium: The Physics of Active Galaxies, ed. G.V. Bicknell, M.A. Dopita, \& P.J. Quinn (San Francisco: Astronomical Society of the Pacific), 367.

Wills, B. J., et al. 1997, this volume. 